\title{
Multifield hybrid approach for bubble to slug flow transition modelling
}

\author{
R. Denefle ${ }^{1}$, S. Mimouni ${ }^{1}$, J.-P. Caltagirone ${ }^{2} \&$ S. Vincent ${ }^{1}$ \\ ${ }^{1}$ EDF R\&D, France \\ ${ }^{2}$ I2M, Université Bordeaux 1, UMR 5295, France
}

\begin{abstract}
This paper aims to assess a conservative interface locating method based on levelset adapted to two fluid model for two phase flow. The objective of the work is twofold: first to check out the prediction of the model on bubble coalescence cases and then to introduce a first description of the multifield hybrid approach for twophase flow modelling. The interface locating method is part of a model dedicated to the simulation of large and distorted bubbles. At different liquid viscosities and densities, the model yields reasonable predictions of terminal velocities and shapes for rising bubble experiments. The overall method relies also on an existing building block, consisting of a set of averaged models dedicated to dispersed bubbles, which has already been validated and has given a reasonable agreement with experimental data in cases where the spherical shape assumption is still valid for the dispersed phase. As a consequence, we present the first step toward a new approach consisting of modelling the small bubbles as a dispersed field and simulating the large and distorted ones.

The main outcome is the simulation of bubble coalescence where the distortion of the interface during the coalescence phenomena is followed. The capability to simulate correctly coalescence phenomena is an important point of the modelling of slug flows with interface locating methods.

Keywords: multifield model, surface tension, interface sharpening, bubble rising, coalescence.
\end{abstract}

\section{Introduction}

Two-phase flows are featuring many industrial applications such as nuclear power plants, heat exchangers and chemical reactors. Based on the interface structure, 
topological classification commonly separates two-phase flow regimes into three main groups. The first group would be separated flows such as annular, film or stratified flows, the second the dispersed flows such as bubbly, droplet or particle flows, and the last group would be the flows such as bubbly annular, churn turbulent or slug flow. Although these regimes have been experimentally confirmed since a few decades [1], their numerical simulation is still challenging and an universal model remains to be established.

There are several approaches for two-phase flow Eulerian modelling, that may be classified by the successive numerical choices, such as the number of fields, the use of space or time averages, the filtering of some space scales, and the way the interfaces are dealt with. As described by Bestion [2], the capability of these approaches to model the different flow regimes are far from being similar. The RANS approach, which is today of widespread use in CFD for industrial applications presents the drawback of filtering every intermittency scales. This means that besides this approach can deals with all flow regimes, it requires a large amount of work on the closure laws models, currently limited to dispersed and separated flows. The DNS and LES without interface filtering are nowadays too expensive to treat high Reynolds number flows such as churn. According to Bestion, an hybrid method so called Hybrid LES, both filtering and simulating interfaces may be a promising way to describe all flow regimes at a reasonable CPU cost without filtering the two-phase structures as RANS approach does.

We present here the first steps building the Hybrid LES method. We propose to treat statistically the interfaces small enough to be considered as spherical, and to simulate the distorted interfaces. This naturally leads to a filter scale imposed by the flow conditions.

This method is implemented in the multifield code NEPTUNE_CFD. The modeling of dispersed phase flows has already been validated [3] and has given a reasonable agreement with experimental data in cases where the spherical shape assumption is still valid for the dispersed phase. As a consequence, we concentrate here our work on the simulation of large and deformed bubbles. A first step toward the Hybrid LES method is to build a set of equations to model the large interface field. Numerical model and its application to bubble rising are summarized here and can be found in [4] for more details.

After proving the efficiency of the method on well-known bubble rising cases, we will test the model on two-bubble coalescence experimental cases. This will comfort the extension of the interface locating model toward slug flows studying.

\section{State of the art for interface treatment and coalescence}

Rise of bubbles in viscous liquids/fluids remains a fundamental problem in fluid physics. Despite several theoretical [5], experimental [6] and over these recent years numerical $[7,8]$ studies, this paradigm is still a topic of major interest.

Most of the current techniques applied to interface flows such as large bubble rising have been developed taking into account two major aspects: 
capturing/tracking the interface and stabilizing the flow solver to handle discontinuous fluid properties at the interface.

Several categories of interface locating exist. The first one is Lagrangian grid methods [9] where the background grid is following the interface. The main limitation is that this method cannot track surfaces that break apart or intersect. The second category is the front tracking method [10]. The motion equation for the flow field is solved on a fixed grid and the interface position is tracked explicitly by markers distributed evenly on the interface. Difficulty generally comes from the repartition of the markers which should ideally be kept equidistant, especially for coalescence of interfaces applications. The third category of these methods consists in capturing the interface using various volume functions defined on the fixed grid used to solve the governing equations of the flow. The volume of fluid [11] method and level-set [12] approaches are parts of this group. VOF methods are conservative, but interface properties such as normal and curvature are difficult to calculate accurately. Level-set methods automatically deal with topological changes but are not conservative. Several techniques have been developed to ensure volume conservation and improve the locating method, such as a combination of Level-set and VOF method [13], or the augmented Lagrangian method coupled to VOF techniques [14].

Olsson and Kreiss [15] introduced a level set method with an artificial compression step performed after the advection of the level set function to ensure that the thickness of the transition layer is preserved.

In our work, the Eulerian approach is use. This approach of the two-fluid model allows the direct use of the volume fraction as an interface function. Thus, the equivalent to the level-set function is self-transported by the resolution of the momentum balance equation. To keep a good location of the interface over time, the same artificial compression step as Olsson's is used.

Concerning coalescence, only few experiments present results on two-bubble coalescence such as Brereton and Korotney's experiment [16]. Numerical works on this phenomena usually follow interface treatment methods and can be found with marker approach, Singh and Shyy [17], and VOF approach, van Sint Annaland et al. [18].

\section{Mathematical formulation and numerical method}

\subsection{Governing equations}

The two-fluid model is based on Eulerian multifield balance equations. Further information can be found in Ishii and Hibiki [19]. The balance equations for isothermal two-fluid model can be written as mass and momentum balances:

$$
\partial_{t}\left(\alpha_{k} \rho_{k}\right)+\partial_{x_{i}}\left(\alpha_{k} \rho_{k} U_{k, i}\right)=\Gamma_{k} \text { with } \sum_{k} \Gamma_{k}=0
$$




$$
\begin{aligned}
\partial_{t}\left(\alpha_{k} \rho_{k} U_{k, i}\right)+\partial_{x_{j}}\left(\alpha_{k} \rho_{k} U_{k, i} U_{k, j}\right)= & \partial_{x_{j}}\left(\alpha_{k} \tau_{k, i j}\right)-\alpha_{k} \partial_{x_{i}}(P) \\
& +\alpha_{k} \rho_{k} g_{i}+I_{k, i}
\end{aligned}
$$

where $\rho_{k}, U_{k}, \alpha_{k}, \Gamma_{k}$ and $\tau_{k, i j}, P$ and $I_{k, i}$ denote respectively the density, the mean velocity, the volumetric fraction, the mass transfer and the total stress tensor including laminar and turbulent contribution, the mean pressure and the interfacial momentum exchange between phases. The interfacial momentum exchange is constituted here by two interfacial forces : surface tension force and drag force. This later appears in momentum balance equations due to averaging. Its local sum over phases is equal to zero and depends on the relative velocity between the phases.

$$
\vec{F}_{d r a g, 1}=\frac{\alpha_{1} \alpha_{2}\left(\overrightarrow{v_{2}}-\overrightarrow{v_{1}}\right)\left(\alpha_{1} \rho_{1}+\alpha_{2} \rho_{2}\right)}{\tau}
$$

As we consider in the paper a local separated flow in the two fluid model, the role of the drag force is to enforce a no slip condition and so equality of the phase velocities at the bubble interface. The closure law, eqn. (3), is applied with a very short relaxation time $\tau$ to achieve large interface $\operatorname{drag}(\tau=\Delta t / 100)$.

\subsection{Surface tension}

Large bubbles are no longer considered as a dispersed, but as a continuous field, therefore the surface tension, eqn. (4), has to be taken into account in the model. From an Eulerian point of view, a surface force has to be implemented in volume:

$$
\vec{F}_{s t a}=\sigma \kappa \vec{n}
$$

where $\sigma, \kappa$ and $\vec{n}$ denote respectively the surface tension, the curvature and the normal vector. The curvature $\kappa$ is defined as the divergence of the unit normal vector at the interface. A color function $c$ locating the interface, such as a level set function, is a descriptor of this normal vector. The unit normal vector and the curvature can be calculated with the color function, obtain by diffusion of the volume fraction. Here it becomes important that the thickness of the interface is kept constant so that the color function gives a good approximation of the local curvature. The Continuum Surface Force (CSF) method described by Brackbill et al. [20] allows the reformulation of the surface tension into an equivalent volume force, eqn. (5), that can be added to the momentum balance equation. In the twofluid model, this force is split between the two phases occupying the cell, since two momentum equations are solved. The average model is here the volume one.

$$
\vec{F}_{s t}^{k}=\alpha_{k} \sigma\left[\nabla \cdot\left(\frac{\nabla c}{\|\nabla c\|}\right)\right] \nabla c d V
$$

\subsection{Interface sharpening}

As already discussed in the previous section, the curvature calculation requires to pay some attention to the interface thickness. Olsson and Kreiss [15] coupled an 
artificial compression method to a level set method to obtain volume conservation. The interface sharpening method consists in resolving the following eqn. (6) on a non physical time step between two physical steps to ensure the interface thickness to be kept constant.

$$
\partial_{\tau}\left(\alpha_{k}\right)+\nabla\left(\alpha_{k}\left(1-\alpha_{k}\right) \vec{n}\right)=\varepsilon \Delta \alpha_{k}
$$

The parameter $\varepsilon$ controls the final interface width at convergence and is here chosen so that the interface thickness is 2 cells. The non-physical time $\Delta \tau$ is chosen to ensure CFL and Fourier numbers under 0.5 and to minimize the number of iteration leading to a steady interface width: $\varepsilon=\Delta x / 2$ and $\Delta \tau=\Delta x / 32$.

\section{Application to bubble rising: Rosant's experiment}

\subsection{Experiment description}

The capturing interface process described in the previous section is validated against the experiments of Raymond and Rosant [6]. Bubbles are released in still water at time $t=0 \mathrm{~s}$. The reference pressure is the atmospheric pressure $1013 h P a$. The fluid viscosity and density (blend of water and glycerol) are given in table 1. The final bubble velocity and shape (ratio between height $h$ and width $w$ of the bubble) are studied as functions of the bubble initial diameter. An axisymmetric geometry is used, reproducing the experiment situation of bubbles rising in a pipe. The air density and viscosity are respectively $1.29 \mathrm{~kg} . \mathrm{m}^{-3}$ and $1.84 * 10^{-5}$ Pa.s. A Dirichlet's condition on the pressure field is imposed at the top boundary.

\subsection{Sensitivity analysis, size of the computational domain}

The experiments were conducted in a rectangular Plexiglas tank of $0.3 \times 0.2 \mathrm{~m}^{2}$ inside cross section and $0.5 \mathrm{~m}$ height. Wall confinement effects on the bubbles can be considered as negligible. As modelling such a huge domain will cost a lot of calculation time, the aim of this analysis is to find the smallest possible computational domain in which walls have negligible effect on the terminal velocity and shape of the simulated bubbles. Radius from 2 to 6 times the bubble

Table 1: Fluid properties for the Raymond and Rosant's experiment [6].

\begin{tabular}{|l|c|c|c|}
\hline Series & Viscosity $[P a . s]$ & Density $\left[\mathrm{kg} / \mathrm{m}^{3}\right]$ & Surface tension $[\mathrm{N} / \mathrm{m}]$ \\
\hline S1 & 0.687 & 1250 & 0.063 \\
\hline S3 & 0.242 & 1230 & 0.063 \\
\hline S5 & 0.0733 & 1205 & 0.064 \\
\hline S6 & 0.0422 & 1190 & 0.064 \\
\hline
\end{tabular}




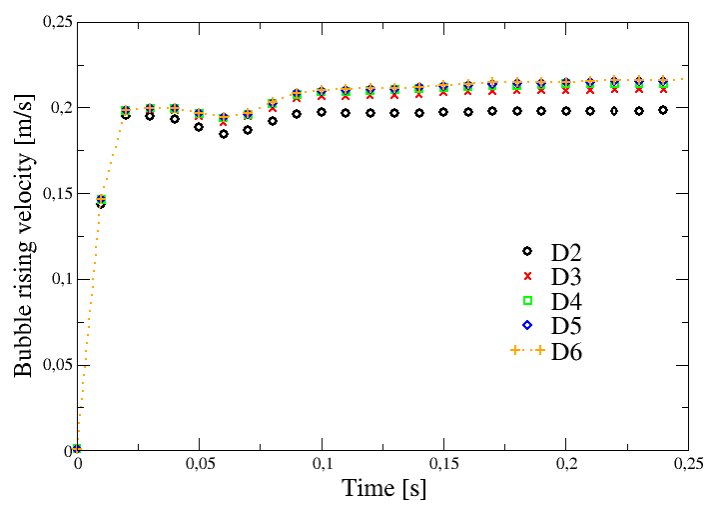

Figure 1: Effect of computational domain size on the bubble shape for an initially $8 \mathrm{~mm}$ diameter spherical bubble in the S5 experiment (see table 1).

diameter were tested for the simulation of the experimental case S5 (see table 1) with an $8 \mathrm{~mm}$ diameter bubble. The fig. 1 presents the terminal bubble shape for the tested computational domain size. The terminal velocity can be considered as independent from the domain size $D$ when $D \leq 4 D_{b}$.

\subsection{Sensitivity to the mesh refinement}

A grid-independent test was carried out on the axisymmetric rise of a single bubble in a liquid on six different meshes. According to the previous sensitivity analysis on the domain size, the domain is chosen to have a four bubble diameter radius, and twelve bubble diameter height. The six grids are M2 $\left(h=D_{\text {bubble }} / \Delta x=15\right)$, M3 $(h=20)$, M4 $(h=25)$, M5 $(h=30)$, M6 $(h=35)$ and M7 $(h=40)$.

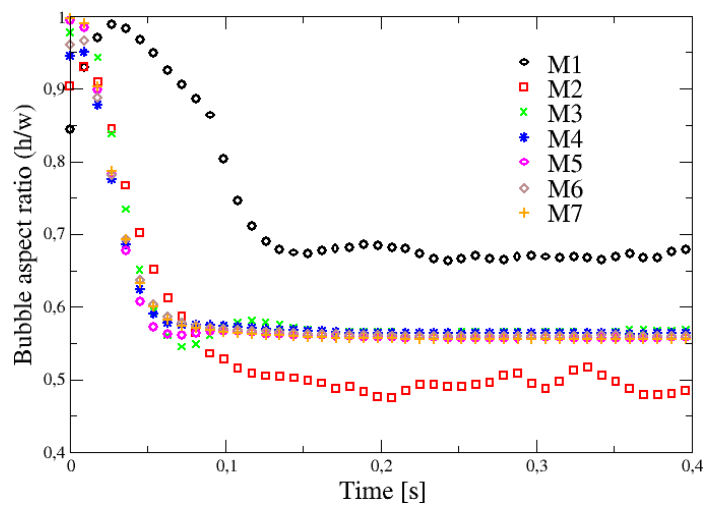

Figure 2: Effect of the mesh refinement on the bubble rising velocity for an initially $8 \mathrm{~mm}$ diameter spherical bubble in the S3 experiment. D is the domain radius given here in number of bubble diameter. 
Fig. 2 shows that the mesh M5 provides a nearly grid-independent solution. This grid will be used in all other runs.

\subsection{Model validation with experiments}

Fig. 3 and 4 present the evolution of terminal velocities and shapes versus the initial bubble diameter for the 4 experiments S1, S3, S5 and S6. The predicted velocities are found to be in a good agreement with the experimental data, the deviation being lower than $10 \%$. Concerning the bubble aspect ratio, the deviation is lower than $10 \%$ for experiment S1 and S3, lower than $23 \%$ for experiment S5 and lower than $30 \%$ for S6. For the most distorted cases (experiment case S6 and bubble diameters greater than $6 \mathrm{~mm}$ ), part of the gap between measures and numerical prediction could be explained by the difficulty to reproduce the exact initial conditions. In the simulation, the bubble is initiated as a still sphere whereas in the experiment the bubble may not be spherical at time $t=0 \mathrm{~s}$, especially for experiments where the bubble is large and the fluid less viscous.

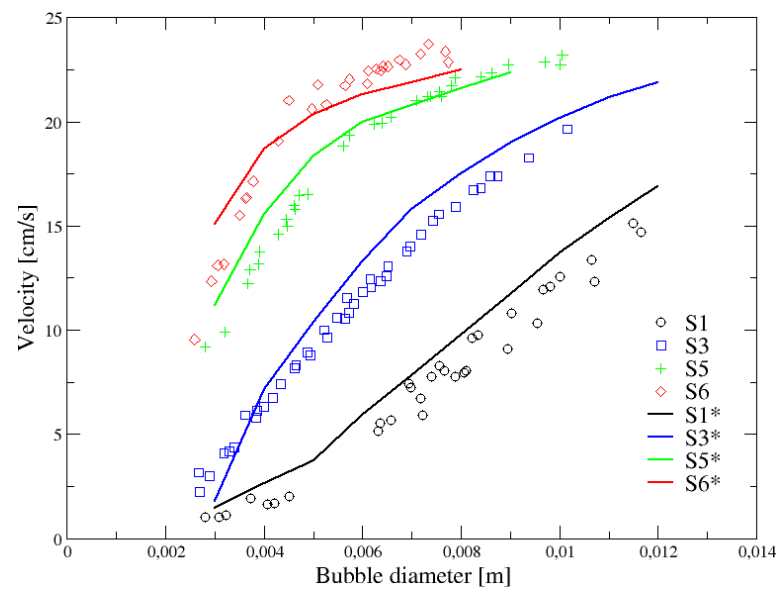

Figure 3: Predicted bubble velocities (solid lines) compared to experimental data (symbols) versus the equivalent diameter for various liquid properties.

\section{Bubble coalescence}

\subsection{Experiment description}

The predictions of the model prove to be in reasonable agreement with the experimental observations for bubble rising cases. For the simulation of slug flows, we need to be able to describe large bubble coalescence phenomena. The experiments of Brereton and Korotney [16] provide a good qualitative description 


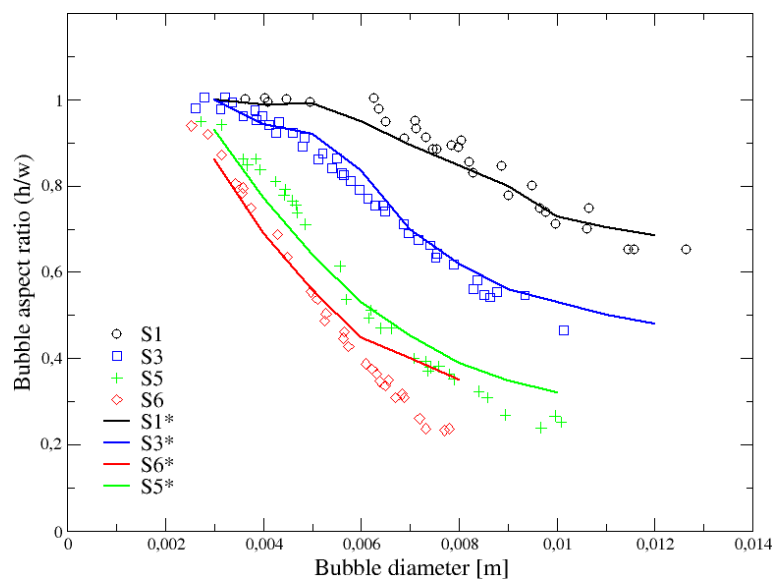

Figure 4: Predicted bubble aspect ratio (solid lines) compared to experimental data (symbols) versus the equivalent diameter for various liquid properties (see table 1).

of the coalescence of two rising bubbles. Four cases are calculated: two cases of coaxial coalescence and two cases of oblique coalescence, each type of coalescence being treated in two dynamic conditions, described by Reynolds, Morton and Eötvos numbers: case $(a),\left(R e=43, M o=2.10^{-4}, E \ddot{o}=16\right)$ and case $(b),(R e=1, M o=30, E \ddot{o}=16)$. The two initial bubbles have the same volume.

\subsection{Results}

Numerical results are presented in figs. 5 and 6 . The computational domain is a rectangular $3 \mathrm{D}$ box of size $0.04 \times 0.04 \times 0.08 \mathrm{~m}^{3}$, the origin is placed at the center of the bottom face. The initial bubble have a radius of $5 \mathrm{~mm}$ and are placed at positions $\{(0,0,0.025)(0,0.08,0.125)\}$ and $\{(0,0,0.025)(0,0,0.125)\}$ respectively for the oblique and the coaxial coalescence experiments. Regarding the discretization, the space scale is $\Delta x=5 \cdot 10^{-3} \mathrm{~m}$ for the case $(a)$ and $\Delta x=2 \cdot 5 \cdot 10^{-3} m$ for the case $(b)$, the fact that the most viscous case need the finest grid is due to the difficulty to reproduce the sharp tail of the second bubble observed on fig. 6 at 0.09 and $0.12 \mathrm{~s}$. The time scale of the coalescence is well predicted by the numerical simulations for all cases, as the time between each experimental photograph is approximatively $30 \mathrm{~ms}$. Despite the difficulty of the phenomena, the shapes of both leading and trailing bubbles are in excellent agreement with the experimental observations. 


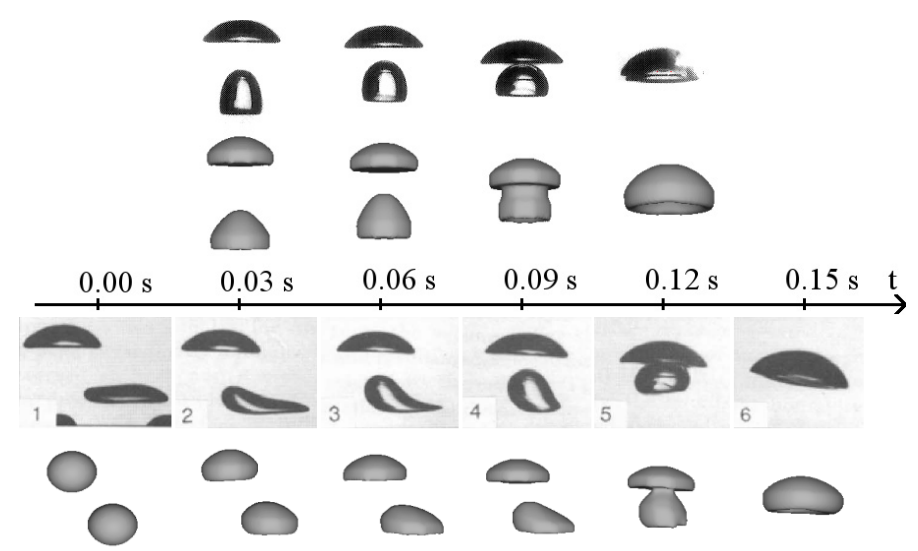

Figure 5: Comparison between the experimental observations from Brereton and Korotney [16] and numerical predictions for coaxial and oblique coalescence of two bubbles: $R e=43, M o=2 \cdot 10^{-4}, E \ddot{o}=16$.

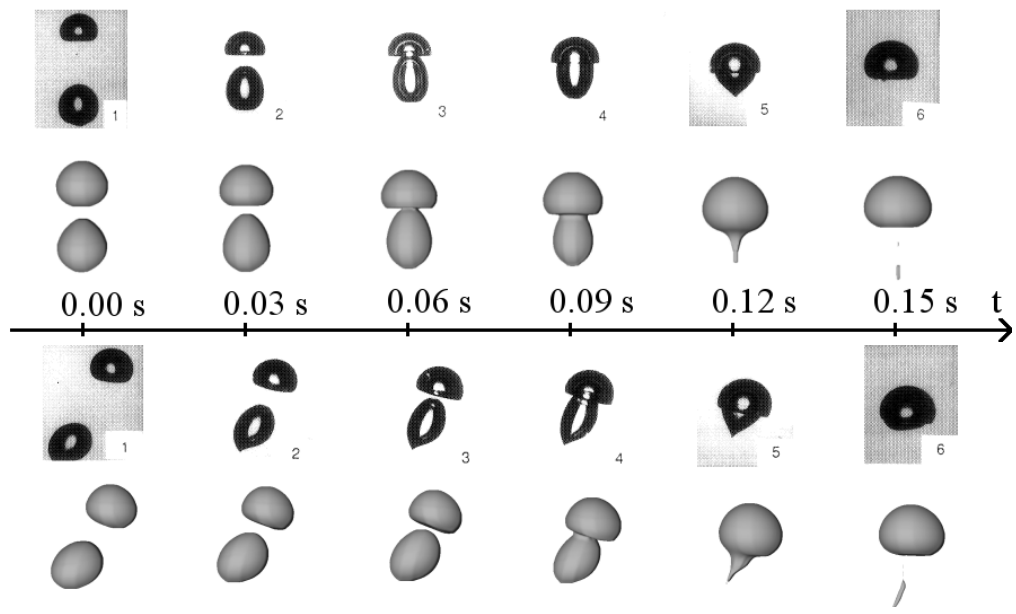

Figure 6: Comparison between the experimental observations from Brereton and Korotney [16] and numerical predictions for coaxial and oblique coalescence of two bubbles: $R e=1, M o=30, E \ddot{o}=16$.

\section{Conclusion}

The dynamics of a deformed bubble has been studied using a two-fluid model adapted to cases with located interfaces. Experimental validations have been carried out. Quantitatively for bubble rising, the predicted terminal shape and velocity of bubbles are in reasonable agreement with the experiments of Raymond and Rosant [6]. Qualitatively for two-bubble coalescence, the coalescence time 
scale and bubble behavior are found to be quite similar to Brereton and Korotney's experiments [16]. The model dedicated to large distorted bubbles was successfully applied to coalescence cases in different liquid conditions. This is comforting us in the way of an hybrid three field model to address regime transitions such as bubbly to slug flow.

\section{Acknowledgements}

This work has been achieved in the framework of the NEPTUNE project, financially supported by CEA (Commissariat à l'Energie Atomique et aux Energies Alternatives), EDF, IRSN (Institut de Radioprotection et de Sûreté Nucléaire) and AREVA-NP, in collaboration with I2M laboratory (Bordeaux, France).

\section{References}

[1] Weisman, J. and Kang, S.Y., Flow pattern transitions in vertical and upwardly inclined lines. International Journal of Multiphase Flow, 7, pp. 271-291, 1981.

[2] Bestion, D., Applicability of two-phase CFD to nuclear reactor thermalhydraulics and elaboration of best practice guidelines. Nuclear Engineering and Design, In Press, p. Corrected proof, 2011.

[3] Mimouni, S., Archambeau, F., Boucker, M., Laviéville, J. and Morel, C., A second order turbulence model based on a reynolds stress approach for two-phase boiling flow and application to fuel assembly analysis. Nuclear Engineering and Design, 240, pp. 2225-2232, 2009.

[4] Denefle, R., Mimouni, S., Caltagirone, J.P. and Vincent, S., Multifield hybrid LES approach for two-phase flow modelling - part 1: Adiabatic bubble rising. Work in progress.

[5] Clift, R., Grace, J.R. and Weber, M.E., Bubbles, drops, and particles. Academic Press, New York, 1978.

[6] Raymond, F. and Rosant, J.M., A numerical and experimental study of the terminal velocity and shape of bubbles in viscous liquids. Chemical Engineering Science, 55, pp. 943-955, 2000.

[7] Hua, J. and Lou, J., Numerical simulation of bubble rising in viscous liquid. Journal of Computational Physics, 222, pp. 769-795, 2007.

[8] Koebe, M., Bothe, D. and Warnecke, H.J., Direct simulation of air bubbles in water/glycerol mixtures : shapes and velocity fields. Proceedings of FEDSM'03: 4th ASME_JSME Joint Fluids Engineering Conference, Honolulu, Hawaii, USA, 2003.

[9] Hyman, J.M., Numerical methods for tracking interfaces. Physica D, 12, pp. 396-407, 1984. 
[10] Unverdi, S.O. and Tryggvason, G., A front-tracking method for viscous, incompressible, multi-fluid flows. Journal of Computational Physics, 100, pp. 25-37, 1992.

[11] Hirt, C.W. and Nichols, B.D., Volume of fluid (VOF) method for the dynamics of free boundaries. Journal of Computational Physics, 39, pp. 201225, 1981.

[12] Osher, S. and Sethian, J.A., Fronts propagating with curvature-dependent speed: Algorithms based on Hamilton-Jacobi formulations. Journal of Computational Physics, 79, pp. 12-49, 1988.

[13] Sussman, M. and Puckett, E., A coupled level set and volume-of-fluid method for computing 3D and axisymmetric incompressible two-phase flows. Journal of Computational Physics, 162, pp. 301-337, 2000.

[14] Vincent, S., Caltagirone, J.P., Lubin, P. and Randrianarivelo, T.N., An adaptive augmented lagrangian method for three-dimensional multimaterial flows. Computers and Fluids, 33, pp. 1273-1289, 2004.

[15] Olsson, E. and Kreiss, G., A conservative level set method for two phase flow. Journal of Computational Physics, 210, pp. 225-246, 2005.

[16] Brereton, G. and Korotney, D., Coaxial and oblique coalescence of two rising bubbles. Proceedings of AMD-Vol. 119: The ASME Applied Mechanics Conference, Columbus, Ohio, USA, 1991.

[17] Singh, R. and Shyy, W., Three-dimensional adaptive grid computation with conservative, marker-based tracking for interfacial fluid dynamics. 44th Aerospace Science Meeting, Reno MV, USA, 2006.

[18] van Sint Annaland, M., Deen, N. and Kuipers, J., Numerical simulation of gas bubbles behaviour using a three-dimensional volume of fluid method. Chemical Engineering Science, 60, pp. 2999-3011, 2005.

[19] Ishii, M. and Hibiki, T., Thermo-fluid Dynamics of Two Phase Flow. Springer-Verlag, 2006.

[20] Brackbill, J., Kothe, D. and Zemach, C., A continuum method for modeling surface tension. Journal of Computational Physics, 100, pp. 335-354, 1992. 